\section{World Trade Center Health Program Providing healthcare to people affected by the September $11^{\text {th }}$ terrorist attacks}

\section{WTC Health Program at a Glance}

The World Trade Center (WTC) Health Program provides specialized medical monitoring and treatment for responders to the September 11, 2001, terrorist attacks in New York City, at the Pentagon, and near Shanksville, Pennsylvania (PA), and for survivors in the New York City Disaster Area. As a result of their exposure to airborne toxins and other hazardous conditions arising from the $9 / 11$ terrorist attacks, responders and survivors now face serious physical and mental health issues, including cancer. Medical benefits are provided to eligible responders and survivors to monitor, evaluate, and treat specific WTC-related health conditions through seven Clinical Centers of Excellence (CCEs) in the New York metropolitan area and through a Nationwide Provider Network (NPN) in all 50 states.

In addition to providing medical care, the WTC Health Program funds medical research to identify new health conditions that may be related to $9 / 11$ exposures and effective treatment for covered health conditions through both individual investigators and the WTC Health Registry, a program of the New York City Department of Health and Mental Hygiene. The WTC Health Program also engages in outreach to people who may be eligible for the Program but have not yet enrolled, and provides education to current members on Program benefits.

The WTC Health Program is administered by the National Institute for Occupational Safety and Health (NIOSH), part of the Centers for Disease Control and Prevention in the U.S. Department of Health and Human Services.

\section{Covered Health Conditions}

The WTC Health Program covers treatment for WTC-related health conditions such as aerodigestive conditions, cancers, mental health conditions, and acute traumatic injuries. As of December 31, 2016, more than 38,700 members were certified for at least one WTC-related health condition, including almost 6,000 members who have at least one WTC-related cancer. Of those 38,700 , more than 28,700 have more than one WTC-related health condition.

The most common covered conditions are:

The most common covered cancers are:
1. Chronic Rhinosinusitis
2. Gastroesophageal Reflux Disease
3. Asthma
4. Sleep Apnea
5. Post-Traumatic Stress Disorder
6. Chronic Respiratory Disorder
7. Cancer
8. Depression
9. Chronic Obstructive Pulmonary Disease
10. Anxiety Disorder

\title{
Facts and Figures
}

- As of January 31, 2017, there were approximately 77,500 members enrolled.

- In 2016, the WTC Health Program enrolled over 3,000 new members.

- The WTC Health Program has members who reside in all 50 states.

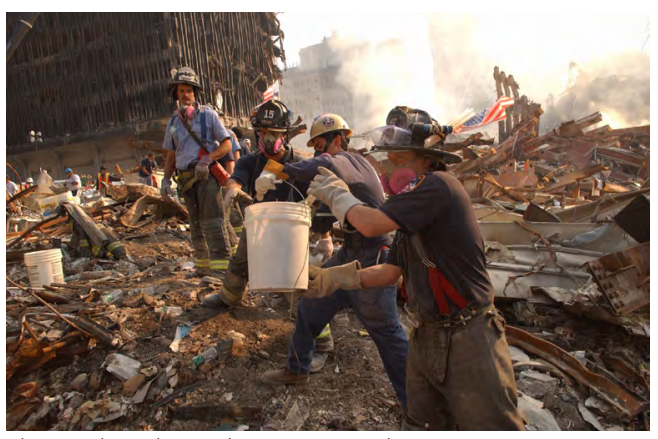

FDNY, 16,907

Photo Credit: Andrea Booher/ FEMA News Photo

DEPARTMENT OF HEALTH AND HUMAN SERVICES

Center for Disease Control and Prevention

National Institute for Occupational Safety and Health

DHHS (NIOSH) Publication No. 2017-141

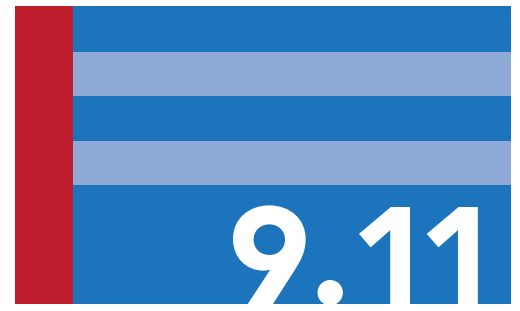

WTC Health Program

\section{Zadroga Act}

The James Zadroga 9/11 Health and Compensation Act of 2010 (Act), which became law in January 2011, established the WTC Health Program and created a fund to pay for the Program. The Act continued previous medical benefits for NYC responders and survivors, expanded benefits to responders at the Pentagon and Shanksville, PA, and established an advisory committee, as well as a research program. The Act also reopened the Department of Justice's September $11^{\text {th }}$ Victim Compensation Fund (VCF).The WTC Health Program works collaboratively with the VCF to ensure consistency between the programs.

\section{Membership}

Program enrollment is based on eligibility criteria established by the Act. These criteria vary based on an individual's activity and location and the duration of their exposure.

Eligible responders include:

- Firefighters

- Police/other law enforcement

- Individuals who performed rescue,

- recovery, cleanup, or support services

Eligible survivors include individuals in the New York City disaster area who were:

- Caught in the dust cloud

- Residents or local workers

- Students or childcare/daycare attendees

\section{Research}

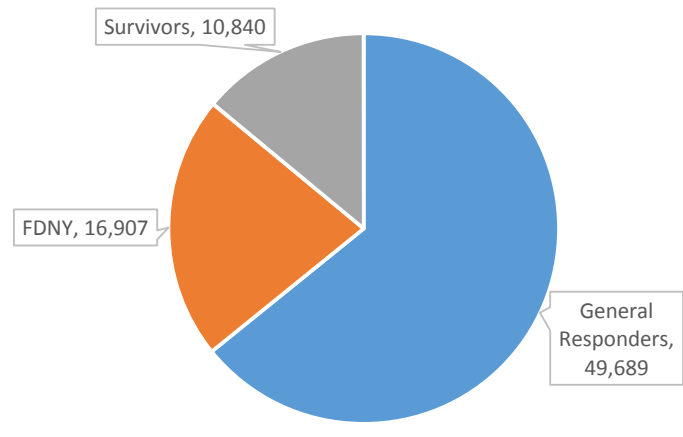

Visit https://www.cdc.gov/wtc/ to learn more exposure-response relationships, and pat-
Each year, the WTC Health Program awards approximately $\$ 15$ million in research designed to help answer critical questions about the physical and mental health conditions related to the 9/11 terrorist attacks. In addition, the Program awards approximately $\$ 7$ million per year to fund the WTC Health Registry that is administered by the New York City Department of Health and Mental Hygiene. Recent funded research topics include biomarkers of exposures or health outcomes, terns of illness (age, gender). To learn more about WTC Health Program-funded research click $\underline{H E R E}$

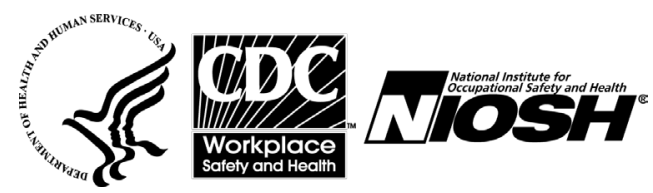

